\title{
Percepción docente sobre la cooperación universitaria al desarrollo en la Universidad Michoacana de San Nicolás de Hidalgo
}

\author{
Teaching Perception of University Development Cooperation at the \\ Universidad Michoacana de San Nicolás de Hidalgo
}

Percepção docente da cooperação para o desenvolvimento universitário na Universidad Michoacana de San Nicolás de Hidalgo

\author{
Rosa Vega Cano* \\ Universidad Michoacana de San Nicolás de Hidalgo, México \\ rosa.vega@umich.mx \\ https://orcid.org/0000-0002-5668-1429 \\ Rogelio Rivera Fernández \\ Universidad de Guadalajara, México \\ rogelioriverafernandez@msn.com \\ https://orcid.org/0000-0003-2123-8360
}

\footnotetext{
*Autor de correspondencia.rosa.vega@umich.mx
}

\section{Resumen}

Aun cuando las universidades han tenido y mantienen una relevancia significativa como fuentes de conocimientos transferibles a la sociedad, los avances alcanzados en términos del desarrollo sostenible son escasos, parcelados y con efectos sinérgicos limitados. Por ello, este trabajo se planteó como objetivo analizar la forma en que se contribuye al desarrollo desde las funciones sustantivas de la Universidad Michoacana de San Nicolás de Hidalgo (UMSNH) mediante la percepción de sus docentes. Se utilizó un enfoque mixto, con la 


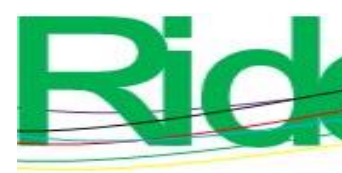

Revista Iberoamericana para la Investigación y el Desarrollo Educativo ISSN 2007 - 7467

improve the conditions of the environment through its teaching, research, extension and dissemination activities; however, these are insufficient and, according to the teachers' own opinion, efforts to generate synergies in favor of sustainable development should be increased in quantity and qualities. Likewise, it was found that university professors assume a responsibility with social development that, from the exercise of their work, they can fulfill. In this sense, the UMNSH is at a key moment that it should take advantage of to strengthen its legitimacy and social relevance.

Keywords: sustainable development, higher education, collaboration networks.

\section{Resumo}

Embora as universidades tenham tido e tenham uma relevância significativa como fontes de conhecimento passíveis de transferência para a sociedade, os avanços em termos de desenvolvimento sustentável são escassos, fragmentados e com efeitos sinérgicos limitados. Portanto, o objetivo deste trabalho foi analisar a forma como o desenvolvimento é contribuído a partir das funções substantivas da Universidade Michoacan de San Nicolás de Hidalgo (UMSNH) por meio da percepção de seus professores. Utilizou-se uma abordagem mista, com o intuito de coletar informações a partir da subjetividade dos professores universitários e, posteriormente, fazer uma análise por meio de técnicas estatísticas que permitissem quantificar as opiniões dos professores de forma geral e por dimensões de análise. Por fim, as informações foram analisadas para identificar possíveis relações entre as variáveis. Portanto, trata-se de um estudo descritivo-correlacional que se apoiou na aplicação de um survey a uma amostra aleatória simples composta por 336 docentes alocados na referida universidade, com representação de todas as áreas do conhecimento. Os resultados permitiram identificar uma percepção muito favorável sobre a cooperação desta universidade para melhorar as condições do meio ambiente por meio de suas atividades de ensino, pesquisa e extensão e divulgação; no entanto, são insuficientes e, na opinião dos próprios professores, os esforços para gerar sinergias em prol do desenvolvimento sustentável devem ser aumentados em quantidade e qualidade. Da mesma forma, constatou-se que o professor universitário assume uma responsabilidade com o desenvolvimento social que, a partir do exercício do seu trabalho, pode cumprir. Nesse sentido, a UMSNH encontra-se em um momento-chave que deve ser aproveitado para fortalecer sua legitimidade e relevância social. 


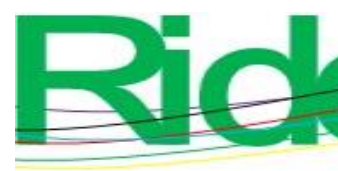

Revista Iberoamericana para la Investigación y el Desarrollo Educativo ISSN $2007-7467$

Palavras-chave: desenvolvimento sustentável, ensino superior, redes de colaboração.

Fecha Recepción: Agosto 2020

Fecha Aceptación: Febrero 2021

\section{Introducción}

El mundo está viviendo una época de profundas y vertiginosas transformaciones en materia económica, social, ambiental, etc., estimuladas por el avance de las tecnologías y por el objetivo (casi imperativo) de la competitividad; todo ello derivado (en gran medida) del proceso de la globalización.

En este contexto, cada vez más complejo, el crecimiento y desarrollo de los países está determinado por una diversidad de factores no tradicionales (como lo eran la inversión, la producción y el consumo), basándose ahora en aspectos como la innovación y el desarrollo tecnológico, la generación y transferencia de conocimiento y la formación de redes locales y globales.

De este modo, las universidades, al ser instituciones en las que convergen las distintas áreas del saber, desde donde se produce, transmite y transfiere conocimiento, se erigen como un actor fundamental en la promoción del desarrollo de los países.

Por otra parte, en este proceso de transnacionalización de las relaciones humanas que se encuentra implícito en el de la globalización, se ha abierto un espacio para el surgimiento de la cooperación entre actores para promover el desarrollo, desde una visión de corresponsabilidad, solidaridad y sostenibilidad en el largo plazo.

Esta idea de la cooperación al desarrollo encuentra un fuerte respaldo en el hecho de que la globalización, pretendiendo ser un proceso homogeneizador (al menos aparentemente), ha derivado en la constitución de un globo completamente heterogéneo en el que la generación e intercambio de conocimientos y tecnologías observa grandes asimetrías que dificultan la incorporación de los menos favorecidos a la lógica de la globalización.

En tales condiciones, se entiende y justifica que las universidades no deben permanecer como espectadoras de los cambios y replicando esquemas que les permiten adaptarse solo de manera parcial y a corto plazo a la lógica predominante; la situación actual demanda de ellas un comportamiento dinámico que ayude a enfrentar la incertidumbre que la complejidad implica, pues en ello radica, en gran medida, su razón de ser. 

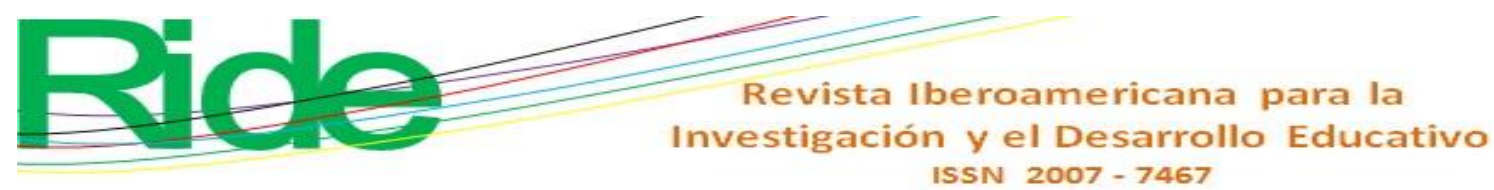

El problema es que, aun cuando las universidades han tenido y mantienen una relevancia significativa como fuentes de conocimientos transferibles a la sociedad, los avances alcanzados en términos de un desarrollo que sea sostenible son escasos, parcelados y con efectos sinérgicos limitados.

Por estas razones, y considerando que la Universidad Michoacana de San Nicolás de Hidalgo (UMSNH) es una universidad pública estatal que cubre la mayor parte de la demanda de educación superior en el estado de Michoacán, este trabajo de investigación planteó como objetivo analizar cómo se contribuye al desarrollo desde sus funciones sustantivas, tomando como marco de referencia los 17 Objetivos de Desarrollo Sostenible (ODS) propuestos desde el seno de la Organización de las Naciones Unidas (ONU) y en particular el ODS 4: Educación de calidad, que busca asegurar que la educación cumpla con tres criterios: inclusión, equidad y calidad, en todos los niveles, en todos los países y a lo largo de la vida de cualquier ciudadano y ciudadana.

Teniendo en cuenta lo anterior, se propuso responder a las siguientes preguntas: 1) ¿la UMSNH está formando ciudadanos capaces de fomentar el desarrollo sostenible?; 2) ¿los conocimientos que esta universidad produce pueden ser aprovechados en la solución de problemas sociales?, y 3) ¿la institución interactúa con otros actores sociales para promover el desarrollo?

Para ello, se definió realizar un estudio de percepción desde la perspectiva docente, dada la diversidad de funciones que asumen los profesores universitarios en el cumplimiento de su labor, ya que, actualmente, no solo se encargan de planear y facilitar el proceso de enseñanza-aprendizaje, sino que realizan investigación, gestionan recursos, se vinculan con el entorno interno y externo a la universidad y, por supuesto, mantienen un contacto directo con la comunidad estudiantil. En ese sentido, se consideró que su opinión en torno al tema podía aportar elementos muy significativos para cumplir con el objetivo de este trabajo.

\section{Elementos de partida sobre la contribución al desarrollo desde las universidades}

El predominio de la concepción neoliberal en las políticas públicas en el mundo propició la mercantilización de la educación superior. Las fuertes presiones financieras a las que fueron sometidas las universidades mediante los recortes presupuestales (que a la fecha prevalecen) condujeron a la adopción de un enfoque que concibe a la educación universitaria 


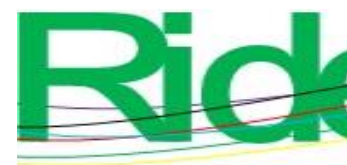

Revista Iberoamericana para la Investigación y el Desarrollo Educativo ISSN $2007-7467$

trabajo altamente calificada, generar lineamientos para la conducción de políticas públicas y promover el pensamiento crítico, la reflexión y la generación de ideas que conlleven al mejoramiento de las condiciones de vida de los habitantes del planeta (Figueroa, 2013), se les atribuye un rol privilegiado en términos de las potencialidades para emprender proyectos y sinergias de gran alcance para lograr la sostenibilidad económica, social y medioambiental.

Estos enfoques alternativos han permeado en la agenda internacional del desarrollo; incluso en el seno de la ONU, ya que en 2015 los países miembros suscribieron una serie de compromisos para fortalecer el combate a la pobreza y a los problemas del medio ambiente desde un enfoque multidimensional, que involucra a las universidades en el cumplimiento de los ODS: 1) Fin de la pobreza, 2) Hambre cero, 3) Salud y bienestar, 4) Educación de calidad, 5) Igualdad de género, 6) Agua limpia y saneamiento, 7) Energía asequible y no contaminante, 8) Trabajo decente y crecimiento económico, 9) Industria, innovación e infraestructura, 10) Reducción de las desigualdades, 11) Ciudades y comunidades sostenibles, 12) Producción y consumo responsables, 13) Acción por el clima, 14) Vida submarina, 15) Vida de ecosistemas terrestres, 16) Paz, justicia e instituciones sólidas y 17) Alianzas para lograr los objetivos (ONU, 2019). Según la Sustainable Development Solutions Network [SDSN] (2017), los ODS "proporcionan una gran oportunidad para mostrar y celebrar el impacto e importancia de lo que las universidades hacen por el bienestar local y global” (p. 40). De ahí que sea posible reenfocar las actividades de las instituciones de educación superior hacia el desarrollo sostenible.

En otras palabras, las universidades se encuentran actualmente de frente a la oportunidad de responder con pertinencia a la sociedad que las creó. Esto mediante la adopción del enfoque de los ODS que incluye diferentes elementos fuertemente interrelacionados: "La reducción de la pobreza, el cambio climático, la reducción de los riesgos de desastre, la biodiversidad, el consumo y la producción sostenibles. [Además] responde a las especificidades locales y respeta la diversidad cultural" (Organización de las Naciones Unidas para la Educación, la Ciencia y la Cultura [Unesco], 2014, p. 33).

Al mismo tiempo, estas instituciones de educación superior tienen un gran reto, ya que asumir esa responsabilidad no solo significa responder, como consecuencia, a las demandas sociales; también representa actuar en coherencia con esa visión desde el interior de sus confines, por lo que el fortalecimiento de las capacidades institucionales juega un papel decisivo para hacer posible esa participación que el desarrollo sostenible demanda de 


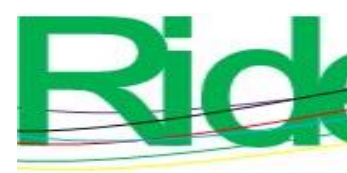

Revista Iberoamericana para la Investigación y el Desarrollo Educativo ISSN 2007-7467

Como puede observarse, las actividades antes mencionadas involucran procesos de formación, investigación, extensión y gestión y, por tanto, a todos los miembros de la comunidad universitaria: estudiantes, docentes, personal administrativo y autoridades (Vallaeys, 2014). La complejidad que implica asumir el compromiso de contribuir al desarrollo sostenible desde las universidades es evidente y llevarlo a la práctica en la realidad, lo es aún más. Sin embargo, también es claro que constituye una posibilidad estratégica para el reencuentro de las universidades con su entorno social. De hecho, diversas universidades han implementado iniciativas innovadoras "para afrontar el reto de conformar una comunidad universitaria integrada por ciudadanos y ciudadanas conscientes de las amenazas socio-ambientales contemporáneas y comprometida en la ardua tarea de superar o minimizar sus consecuencias a niveles local, regional y global” (González, Meyra y Martínez, 2015, p. 89). De lo dicho hasta aquí se puede inferir que la universidad es un detonante del cambio de conciencia social de la sostenibilidad.

Es importante considerar que los enfoques aquí descritos coexisten en la realidad actual y generan resultados igualmente contradictorios al interior de las universidades, pues algunas de estas instituciones que han buscado implementar programas sostenibles no han logrado avances significativos ni estructurales en su interior y tampoco han logrado constituir modelos de referencia para la sociedad en la que se desenvuelven. En ese sentido, "la definición retórica acaba por estrellarse contra barreras e inercias sociales, económicas y académicas, fuertemente establecidas, que impiden avances reales y significativos" (González et al., 2015, p. 78). Y en consecuencia, el cambio en las universidades es un camino a largo plazo.

A partir de lo anteriormente expuesto, es difícil encontrar y denominar una predominancia nítida indiscutible en las formas en las que la universidad se relaciona con su entorno social. Existen en la actualidad formas híbridas que conviven y frecuentemente se yuxtaponen (o se acomodan, bloquean y restringen) entre ellas, condicionando las posibilidades de dar continuidad a ciertos procesos. Un reto adicional es, por tanto, impedir que los roces y fricciones que naturalmente se producen en esa hibridación bloqueen el surgimiento de iniciativas innovadoras capaces de transformar a la universidad y a la sociedad actual. 


\section{Materiales y método}

En esta investigación se trabajó desde un enfoque mixto, con la intención de recabar información basada en la subjetividad de los profesores universitarios, así como en su experiencia profesional en la docencia en el ámbito universitario (Núñez, 2017), para posteriormente realizar un análisis mediante técnicas estadísticas que permitieran cuantificar las opiniones de los docentes de forma general y por dimensiones de análisis, y así identificar tanto una valoración a escala global como a nivel dimensional en torno a la percepción de los docentes sobre la contribución al desarrollo a través de las funciones sustantivas universitarias. Finalmente, se analizó la información para identificar posibles relaciones entre variables.

Por tanto, se trata de un estudio descriptivo-correlacional que se apoyó en la aplicación de una encuesta dirigida al personal docente de la UMSNH, tomando como guía un instrumento desarrollado por Vallaeys, De la Cruz y Sasia (2009) y actualizado por Vallaeys (2014), a partir del cual se analizó la información en 2019. Dicho instrumento tiene por objeto identificar la forma en que las instituciones de educación superior "responden a la sociedad mediante el desarrollo de su misión, y cómo pueden incorporar medidas que permitan mejorar y hacer significativa su contribución para una sociedad más justa y sostenible" (Vallaeys et al., 2009, p. 1).

El cuestionario aplicado fue adaptado y ajustado a las características de este trabajo de investigación. El instrumento final incluyó un total de 53 ítems referidos a diversas variables y categorías de análisis: 1) Edad, sexo, máximo grado académico, dependencia de adscripción y situación contractual del personal docente; 2) Marco normativo, de planeación y organizacional actual de la institución; 3) Actividades de formación, investigación y extensión y difusión; 4) Financiamiento institucional para actividades orientadas a contribuir al desarrollo, y 5) Instrumentos externos de apoyo.

La dimensión "Marco normativo..." incluyó ítems para reflexionar acerca de los contenidos de la Ley Orgánica del Estatuto Universitario, del Plan de Desarrollo Institucional y del organigrama formal de la UMSNH, pues se considera que estos documentos pueden reflejar el grado de compromiso que la institución asume con la cooperación para el desarrollo al integrarla desde la normatividad, las estrategias de acción y la estructura organizacional que orientan su funcionamiento y operación. 


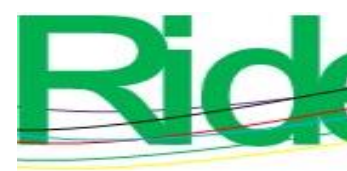

Revista Iberoamericana para la Investigación y el Desarrollo Educativo ISSN 2007 - 7467

de maestría o especialidad, $28 \%$ con doctorado y $25 \%$ con licenciatura; $72 \%$ eran profesores definitivos (ya sea de tiempo completo o parcial), $22 \%$ profesores interinos y $6 \%$ profesores jubilados.

Todas las áreas del conocimiento quedaron representadas en la muestra: $27 \%$ de docentes del área de ciencias de la salud, $22 \%$ de ingenierías y arquitectura, $17 \%$ de ciencias económico-administrativas, $12 \%$ de derecho, $10 \%$ de ciencias biológico-agropecuarias, $7 \%$ de humanidades y $5 \%$ de ciencias exactas, metalurgia y materiales.

El análisis de la información se realizó mediante la estimación de frecuencias (Rustom, 2012), codificación de escalas para identificar la percepción global y por dimensiones en torno a la cooperación para desarrollo (Hernández, Fernández y Baptista, 2014); finalmente, se estimaron correlaciones a través del coeficiente de contingencia y el coeficiente de correlación de Spearman, así como nivel de significancia (Wackerly, Mendenhall y Scheaffer, 2010).

\section{Resultados}

\section{Análisis de frecuencias}

La información recopilada indicó que la mayoría de docentes conocía solo de manera parcial la normativa universitaria (53\%), el plan de desarrollo institucional (51\%) y la estructura organizacional (48 \%); sin embargo, $89 \%$ manifestó estar de acuerdo (total o parcialmente) en que dicho marco institucional debería establecer el compromiso de la UMSNH con el desarrollo desde su Ley Orgánica y el Estatuto Universitario; $91 \%$ en que deberían definirse líneas de acción, estrategias, proyectos específicos orientados a procurar el mejoramiento de las condiciones económicas, sociales y ambientales de nuestro entorno, y $88 \%$ en que deberían asignarse responsables institucionales para este tipo de proyectos o estrategias, así como reconocérseles dentro del organigrama institucional.

En cuanto a las actividades de formación que se realizan en esta universidad, la mayoría de los docentes consideró que la educación que brinda la UMSNH debería orientarse a la formación de profesionales comprometidos con el desarrollo sostenible (94\%) mediante la oferta de un mayor número de cursos o asignaturas con contenidos que coadyuven a crear conciencia social en la comunidad universitaria (91\%), así como a través de la creación de programas de servicio social o voluntariado solidario en los que se involucre a los estudiantes 


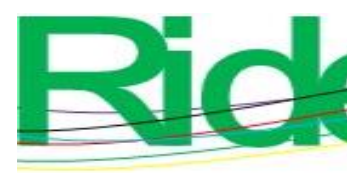

Revista Iberoamericana para la Investigación y el Desarrollo Educativo ISSN 2007-7467

En el mismo orden de ideas, $89 \%$ considera que, aun cuando la UMSNH cuenta con líneas de investigación orientadas al desarrollo, estas deben ser fortalecidas (78 \%), procurando que los proyectos sean definidos en consulta con actores externos con la intención de atender problemáticas o demandas sociales específicas (79\%) y de manera interdisciplinaria $(71 \%)$. Aproximadamente $30 \%$ de los docentes no ha participado en investigaciones de este tipo.

Asimismo, $88 \%$ de los docentes estima importante que esta universidad suscriba convenios de colaboración con otros actores clave del desarrollo (gobiernos, organizaciones no gubernamentales, organismos internacionales y empresas), organice y lleve a cabo eventos como charlas, debates, congresos, simposios, cursos, publicaciones, entre otros, con el fin de sensibilizar a la comunidad académica y a la sociedad en general en temas específicos del desarrollo (88\%).

Es importante destacar que $77 \%$ de los docentes manifestó estar de acuerdo con afirmaciones que señalan que en esta universidad se organizan actividades de extensión y difusión de este tipo, por medio de las cuales se logra vincular la enseñanza y la investigación con las necesidades sociales $(90 \%)$ y brindar a sus estudiantes y docentes oportunidades de interacción con diversos actores sociales $(77.9 \%)$. Sin embargo, poco más de la cuarta parte de encuestados $(27 \%)$ respondió que no ha podido formar parte de grupos o redes con fines sociales organizados o promovidos por la universidad.

Atendiendo al tema del financiamiento para actividades de cooperación al desarrollo, la mayoría de los docentes expresó estar totalmente de acuerdo en que es importante que la institución destine recursos financieros para llevar a cabo actividades para contribuir al desarrollo (65\%) e incluso en que se destine una mayor cantidad de ellos para la realización de este tipo de actividades (84 \%). No obstante lo anterior, $39 \%$ de los profesores manifestó no haberse involucrado en este tipo de actividades

Igualmente, los docentes expresaron estar de acuerdo en que es importante que la universidad haga uso de instrumentos (programas, proyectos y acciones específicas) que ofrecen redes, asociaciones y organismos nacionales e internacionales para extender las posibilidades de acción y contribuir a mejorar las condiciones de vida de la población (93 \%), pero $49 \%$ declaró no haber participado en actividades de esta naturaleza. 


\section{Valoración de la cooperación al desarrollo}

Para identificar la percepción que tienen los docentes en torno a la cooperación para el desarrollo que realiza la UMSNH, se asignó una puntuación a las respuestas obtenidas en relación con el puntaje que podía obtenerse en total por todos los ítems contenidos en la encuesta y se creó una nueva escala de valoración, a partir de intervalos específicos, de acuerdo con el puntaje total. De esta manera, fue posible identificar tanto la percepción global como por dimensiones y categorías de análisis. Los resultados recuperados se muestran en la tabla 1.

Tabla 1. Percepción de los docentes en torno a la cooperación universitaria al desarrollo, según dimensión, categoría de análisis y global

\begin{tabular}{|l|c|c|c|c|c|}
\hline \multirow{2}{*}{ Percepción CUD } & \multicolumn{5}{|c|}{ Valoración (frecuencia \%) } \\
\cline { 2 - 6 } & $\begin{array}{c}\text { Muy } \\
\text { desfavorable }\end{array}$ & Desfavorable & Regular & Favorable & $\begin{array}{c}\text { Muy } \\
\text { favorable }\end{array}$ \\
\hline Global & - & - & $5 \%$ & $39 \%$ & $56 \%$ \\
\hline Marco institucional & - & $1.2 \%$ & $4.5 \%$ & $38.1 \%$ & $56.3 \%$ \\
\hline Normatividad & $0.6 \%$ & $2.7 \%$ & $10.1 \%$ & $33.6 \%$ & $53 \%$ \\
\hline Planeación & $0.3 \%$ & $2.1 \%$ & $14.6 \%$ & $43.2 \%$ & $39.9 \%$ \\
\hline Estructura & $1.2 \%$ & $3.3 \%$ & $8.6 \%$ & $38.4 \%$ & $48.5 \%$ \\
\hline organizacional & & & & & \\
\hline Actividades & - & - & $5.7 \%$ & $39.9 \%$ & $54.5 \%$ \\
\hline Formación & - & $0.6 \%$ & $4.2 \%$ & $27.4 \%$ & $67.9 \%$ \\
\hline Investigación & - & $1.5 \%$ & $11.9 \%$ & $41.4 \%$ & $45.2 \%$ \\
\hline Extensión y difusión & - & $1.2 \%$ & $12.8 \%$ & $41.7 \%$ & $44.3 \%$ \\
\hline Financiamiento & $0.6 \%$ & $4.8 \%$ & $9.8 \%$ & $41.7 \%$ & $43.2 \%$ \\
\hline Instrumentos externos & $0.6 \%$ & $3 \%$ & $22.6 \%$ & $33 \%$ & $40.8 \%$ \\
\hline
\end{tabular}

Fuente: Elaboración propia

Como puede observarse, la valoración global es muy favorable; sin embargo, es necesario acercar la mirada al interior de las dimensiones, pues se observa que, en diversas categorías, no hay una diferencia muy significativa entre el porcentaje de docentes que tiene una opinión muy favorable y los que tienen una valoración solo favorable. 


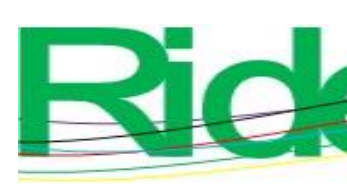

Revista Iberoamericana para la
Investigación y el Desarrollo Educativo
ISSN $2007-7467$

Dentro de "Marco normativo...", esta situación puede obedecer a que quienes tienen una percepción muy favorable es porque la sustentan en las potencialidades que consideran puede ofrecer un marco normativo, de planeación y de organización que se comprometa explícitamente con la cooperación al desarrollo; sin embargo, debe tenerse en cuenta que una importante proporción de docentes no conoce (o no del todo) estos documentos normativos, de planeación y de organización.

Respecto a la dimensión "Actividades...", es importante recordar que incluye ítems orientados a la reflexión de los docentes en torno a lo que consideran el deber ser en términos del cumplimiento de las funciones sustantivas universitarias, así como aspectos en los cuales los encuestados están implicados directamente en su quehacer cotidiano como miembros de la comunidad académica, de lo que puede deducirse que los docentes observan que es necesario incrementar y fortalecer estrategias que permitan integrar la dimensión social de forma transversal en la formación, investigación y extensión y difusión para ofrecer una educación más integral a sus estudiantes.

En las dimensiones "Financiamiento..." e "Instrumentos externos de apoyo", los participantes legitiman, al mostrar una percepción muy favorable, la disposición de recursos monetarios para la realización de actividades de cooperación al desarrollo y otorgan importancia a los esfuerzos institucionales que realiza la universidad para acercarse a entes públicos y privados, nacionales e internacionales y coordinar con ellos proyectos que le permitan ampliar sus posibilidades para realizar actividades de cooperación al desarrollo. Esta percepción de los docentes puede explicarse por el papel que ellos mismos tienen en la conformación de redes de colaboración y en la ejecución y operación de programas y proyectos que se establecen institucionalmente.

\section{Análisis de las correlaciones}

Las correlaciones significativas para los datos recuperados de la encuesta a docentes son algunas bajas (cuyos valores oscilan entre 0.200 y 0.399 ) y otras moderadas (entre 0.400 y 0.599).

En la dimensión "Marco normativo..." todos los coeficientes son bajos, lo que supone que la percepción general que tienen los docentes sobre la cooperación al desarrollo no está influenciada por el conocimiento que tienen de dicho marco o la opinión que tienen en 


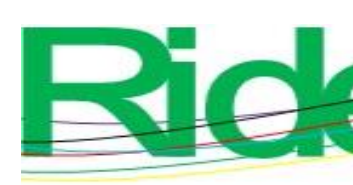

Revista Iberoamericana para la Investigación y el Desarrollo Educativo ISSN 2007-7467

relación con que este debe contener (o no) líneas definidas sobre el compromiso que asume la universidad con el desarrollo (y viceversa).

En la dimensión "Actividades..." existen correlaciones tanto bajas como moderadas, sin embargo, puede decirse, con $99 \%$ de certeza, que si los docentes están adscritos a programas que incluyen asignaturas relacionadas con temas del desarrollo tendrán una percepción muy favorable de la cooperación al desarrollo; lo mismo ocurre si, dentro de sus cursos, promueven la participación de los alumnos en proyectos sociales fuera de la universidad, vinculan la enseñanza con los problemas sociales y promueven que los alumnos tengan contacto directo con especialistas en temas de desarrollo; o bien si perciben que la universidad se preocupa por los problemas sociales y promueve que los estudiantes se involucren en actividades que contribuyan al desarrollo.

Finalmente, la única correlación significativa que existe, en términos de la percepción global de la cooperación al desarrollo, es entre esta y el área del conocimiento a la que corresponde la dependencia de adscripción del docente, entre las que destacan las ciencias de la salud, las ciencias económico-administrativas y el derecho.

\section{Discusión}

De manera general, puede señalarse que prevalece una percepción muy favorable entre los docentes de la UMSNH en cuanto la cooperación al desarrollo, tanto en términos conceptuales (el deber ser) como en el terreno de la práctica de esta institución de educación superior (los hechos), aunque se reconoce la necesidad de incrementar los esfuerzos y capacidades institucionales para tener impactos más significativos sobre la construcción de una sociedad más justa y equitativa. Esto concuerda con lo señalado por González et al. (2015) sobre la existencia de barreras e inercias sociales, económicas y académicas que aún se encuentran fuertemente establecidas en las universidades, que limitan lograr impactos de mayor alcance.

Por otra parte, este hallazgo da cuenta de que existe un grado importante de sensibilización social, en términos generales, en la planta docente de la UMSNH, además de un significativo interés por involucrarse en actividades de esta naturaleza, lo cual lleva a pensar que también hay un importante nivel de consciencia del papel que pueden asumir los profesores como agentes protagónicos del desarrollo tanto desde el ejercicio de su profesión como en las actividades que realizan en el aula. 


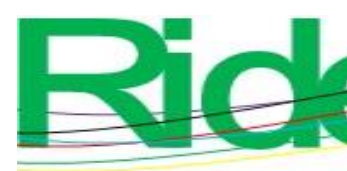
Revista Iberoamericana para la
Investigación y el Desarrollo Educativo
ISSN $2007-7467$

proyectos de cooperación al desarrollo, capacitación para que desarrollen conocimientos, competencias, actitudes y valores.

Aunado a ello, la implicación del estudio para la educación superior se centra en la percepción del profesor como agente del cambio del desarrollo sostenible, lo cual también concuerda con lo expuesto por la Unesco $(2017$, p. 7) en el sentido de que la educación superior puede proporcionar el conocimiento, la conciencia y la acción que empoderen a las personas, en este caso a los profesores, para transformarse en primer lugar a sí mismos y, con ello, transformar la sociedad (Unesco, 2020).

Asimismo, la investigación evidencia que, si bien la educación enfocada al desarrollo sostenible implica la integración de tres dimensiones en el proceso de enseñanza-aprendizaje por parte de las universidades: una dimensión cognitiva, una dimensión socio-emocional y una dimensión conductual, como sostiene una vez más la Unesco (2019), también es necesario que el profesor participe y se involucre de forma activa en las acciones que conlleva cada dimensión; de lo contrario, el desarrollo sostenible se encontrará en los planes y programas de la universidad pero no se materializará en cambios individuales de los docentes y, por ende, no se alcanzará el desarrollo sostenible.

\section{Conclusiones}

El contexto local y global actual está haciendo un llamado urgente para que las universidades participen de forma dinámica y activa en la resolución de problemáticas económicas, sociales y ambientales, propios del desarrollo sostenible. Esto debe ser entendido y aprovechado por las universidades para fortalecer o consolidar su legitimación y pertinencia social.

Debe reconocerse que la cooperación universitaria al desarrollo es una labor compleja y como tal, enfrenta ciertos retos para lograr resultados duraderos y de gran impacto. Entre estos retos se encuentran, por una parte, lograr el involucramiento de todos los actores que integran la comunidad universitaria y, por la otra, la construcción de un marco de fomento y financiamiento tanto desde las mismas universidades como por parte de los demás actores que influyen en el ámbito de la educación superior y, también, es necesario que se le reconozca formalmente en las agendas universitarias.

Los resultados de esta investigación permiten responder de la siguiente manera a las preguntas orientadoras: la percepción de los docentes de la UMSNH en torno a la 


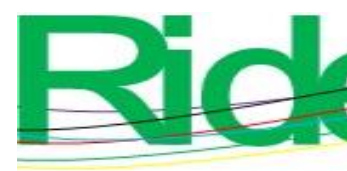

Revista Iberoamericana para la Investigación y el Desarrollo Educativo ISSN 2007 - 7467

contribución al desarrollo que se realiza desde esta institución es, en general, muy favorable y significa, en suma, que esta universidad está formando ciudadanos capaces de fomentar el desarrollo sostenible, que los conocimientos que en ella se producen pueden ser aprovechados en la solución de problemas sociales y que la institución interactúa con otros actores sociales para promover el desarrollo. Sin embargo, también se identificó que los docentes consideran que las actividades y los logros alcanzados por estas, a la fecha, no son suficientes, por lo que los esfuerzos por generar sinergias a favor del desarrollo sostenible deben ser incrementados en cuantía y cualidades.

Asimismo, se encontró que los profesores universitarios asumen una responsabilidad con el desarrollo social que, desde el ejercicio de su labor, pueden cumplir, ya sea promoviendo entre sus estudiantes la formación de una conciencia crítica y comprometida con el entorno que le rodea, o bien gestionando y promoviendo trabajos de investigación que sean pertinentes socialmente.

Finalmente, puede señalarse que la UMSNH se encuentra en un momento clave que debe aprovechar para fortalecer su legitimidad y pertinencia social, asumiendo, cuando menos, algunas políticas compensatorias (o reequilibradoras) destinadas a contribuir en la solución de problemáticas sociales específicas y desarrollando estrategias más amplias e incluyentes, que permitan su acercamiento con el entorno social a través del diseño y puesta en marcha de programas y proyectos que logren vincularla con el resto de actores (locales e internacionales) implicados en los procesos de desarrollo de manera sostenible.

Igualmente, se esboza la necesidad de superar los planteamientos que, aun siendo relativamente más avanzados respecto a los modelos tradicionales, continúan en parte reduciéndose a esquemas aislados y dispersos de colaboración, solamente puntual con otros actores, pues, en algunos de ellos, se sobrevaloran las posibilidades de que el establecimiento de redes de colaboración implique (necesariamente) mejoras en la calidad de vida, e incluso el inicio de procesos de crecimiento económico, puesto que, aun existiendo redes de colaboración (de alta cooperación y participación comunitaria), no siempre consiguen generar dinámicas de desarrollo y obtienen solo ciertas mejoras parciales y transitorias que no alcanzan el involucramiento o la asimilación requeridos para convertirse en verdaderos enlaces sinérgicos para el desarrollo integral y sostenible.

Para culminar estas reflexiones finales, es importante enfatizar en que el entorno actual demanda que las universidades adopten el enfoque de los ODS de manera urgente y 


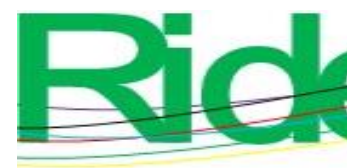

Revista Iberoamericana para la
Investigación y el Desarrollo Educativo
ISSN $2007-7467$

que reflexionen en torno a la contribución que realizan en la construcción de una sociedad global más justa, equitativa, pacífica y tolerante, principalmente desde su función formativa, no únicamente a partir del alumno, sino también del profesor como agente de cambio social, pues el docente es el vínculo que genera sinergias entre la universidad, el alumno y la sociedad.

\section{Futuras líneas de investigación}

Conforme a las tendencias prospectivas de la Red Española para el Desarrollo Sostenible (2020a, 2020b), y según lo expuesto en esta investigación, algunas futuras líneas de investigación podrían ser: 1) el estudio de las competencias universitarias para el cumplimiento de los ODS, 2) el compromiso de la universidad para la sociedad en el marco del desarrollo sostenible, 3) la creación, transferencia y aplicación del conocimiento en las instituciones de educación superior en el marco de los ODS y 4) el impacto de las funciones sustantivas de las instituciones de educación superior para el cumplimiento los ODS.

\section{Referencias}

Aguilar, M., Crisanto, O. y Sánchez, G. (2013). Educación superior y desarrollo en América Latina: un vínculo en debate. ¿La necesaria mediación del mercado? En Martínez, M. E., Piñero, F. J. y Figueroa, S. A. (coords.), El papel de la universidad en el desarrollo (pp. 23-44). México: Benemérita Universidad Autónoma de Puebla-Universidad Nacional del Centro de la Provincia de Buenos Aires. Recuperado de https://www.google.com.mx/url?sa=t\&rct=j\&q=\&esrc=s\&source=web\&cd=\&ved= 2ahUKEwjis435rZ7sAhU9AZ0JHbFWA5YQFjAAegQIAxAC\&url=http\%3A\%2F \%2Fbiblioteca.clacso.edu.ar\%2Fclacso\%2Fse\%2F20140211121020\%2Funiversidad .pdf\&usg=AOvVaw3TFjDn6ZhxD7EwplyTX4Mg.

Alonso, J. A. (2010). La universidad como agente de cooperación al desarrollo: algunas consideraciones generales. En Arias, S. y Molina, E. (coord. ${ }^{\text {as }}$ ). Universidad y cooperación al desarrollo (pp. 29-35). España: Universidad Autónoma de Madrid. Recuperado de http://biblioteca.hegoa.ehu.es/registros/author/7999.

Álvarez, S. (2012). Una introducción a la cooperación internacional al desarrollo. Revista Electrónica de Derecho de la Universidad de La Rioja, (10), 285-309. Recuperado de https://dialnet.unirioja.es/descarga/articulo/4104832.pdf. 


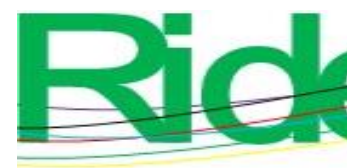

Revista Iberoamericana para la Investigación y el Desarrollo Educativo ISSN $2007-7467$

Figueroa, V. (2013). El rol de la universidad en el desarrollo. La perspectiva de los organismos internacionales. En Martínez, M. A., Piñero, F. J. y Figueroa, S. A. (coords.), El papel de la universidad en el desarrollo (pp. 11-22). Recuperado de https://www.google.com.mx/url?sa=t\&rct=j\&q=\&esrc=s\&source=web\&cd=\&ved= 2ahUKEwjis435rZ7sAhU9AZ0JHbFWA5YQFjAAegQIAxAC\&url=http\%3A\%2F \%2Fbiblioteca.clacso.edu.ar\%2Fclacso\%2Fse\%2F20140211121020\%2Funiversidad .pdf\&usg=AOvVaw3TFjDn6ZhxD7EwplyTX4Mg.

García, C. (2018). La mercantilización de la educación superior en Colombia. Revista Educación y Humanismo, 20(34), 36-58. Recuperado de http://revistas.unisimon.edu.co/index.php/educacion/article/view/2857.

Gómez, C. (2016). El desarrollo sostenible: Conceptos básicos, alcance y criterios para su evaluación. Organización de las Naciones Unidas para la Educación, la Ciencia y la Cultura. Recuperado de https://www.rua.unam.mx/portal/recursos/ficha/83568/eldesarrollo-sostenible-conceptos-basicos-alcance-y-criterios-para-su-evaluacion.

González, E., Meyra, P. y Martínez, C. (2015). Sustentabilidad y universidad: retos, ritos y posibles rutas. Revista de la Educación Superior, 44(175), 69-93. Recuperado de http://www.scielo.org.mx/scielo.php?pid=S018527602015000300004\&script=sci_abstract.

Hernández, R., Fernández, C. y Baptista, P. (2014). Metodología de la investigación. Ciudad de México, México: McGraw Hill.

Llanos, M. y Monzó, J. (2010). Universidad y cooperación para el desarrollo. En Calabuig, C. y de los Llanos, M. (coord. ${ }^{\text {as }}$ ), La cooperación internacional para el desarrollo (pp. 117-131). España: Editorial Universitat Politècnica de València. Recuperado de http://www.upv.es/upl/U0566378.pdf.

Núñez, J. (2017). Los métodos mixtos en la investigación en educación: hacia un uso reflexivo. Cuadernos de Pesquisa, 47(164), 632-649.

Organización de las Naciones Unidas [ONU]. (2019). Agenda 2030 y los Objetivos de Desarrollo Sostenible. Una oportunidad para América Latina y el Caribe. Santiago, Chile: Organización de las Naciones Unidas. Recuperado de https://www.cepal.org/es/publicaciones/40155-la-agenda-2030-objetivos-desarrollosostenible-oportunidad-america-latina-caribe. 


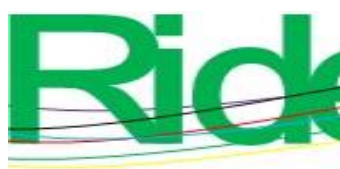

Revista Iberoamericana para la Investigación y el Desarrollo Educativo ISSN 2007 - 7467

Organización de las Naciones Unidas para la Educación, la Ciencia y la Cultura [Unesco]. (2014). Hoja de ruta para la ejecución del Programa de acción mundial de Educación para el Desarrollo Sostenible. París, Francia: Organización de las Naciones Unidas para la Educación, la Ciencia y la Cultura. Recuperado de https://unesdoc.unesco.org/ark:/48223/pf0000230514_spa.

Organización de las Naciones Unidas para la Educación, la Ciencia y la Cultura [Unesco]. (2017). Educación para los Objetivos de Desarrollo Sostenible. Objetivos de aprendizaje. París, Francia: Organización de las Naciones Unidas para la Educación, la Ciencia $\quad y \quad$ la Cultura $\quad$ Recuperado de https://unesdoc.unesco.org/ark:/48223/pf0000252423.

Organización de las Naciones Unidas para la Educación, la Ciencia y la Cultura [Unesco]. (2020). Educación para el desarrollo sostenible: hoja de ruta. París, Francia: Organización de las Naciones Unidas para la Educación, la Ciencia y la Cultura. Recuperado de https://unesdoc.unesco.org/ark:/48223/pf0000374802?posInSet=2\&queryId=N3cced673-a9dc-4d9b-abfe-9e42ca10edb2.

Red Española para el Desarrollo Sostenible [REDS]. (2020a). Cómo evaluar los ODS en las universidades. España: Red Española para el Desarrollo Sostenible. Recuperado de https://reds-sdsn.es/wp-content/uploads/2020/04/Gui\%CC\%81a-COMOEVALUAR-ODS-2020-AAFF.pdf.

Red Española para el Desarrollo Sostenible [REDS]. (2020b). Implementando la Agenda 2030 en la universidad. Casos inspiradores de educación para los ODS en las universidades españolas. España: Red Española para el Desarrollo Sostenible. Recuperado de https://reds-sdsn.es/wp-content/uploads/2020/05/DosierREDS_Casos-ODS-Univ-2020_web-1.pdf.

Rustom, A. (2012). Estadística descriptiva, probabilidad e inferencia. Una visión conceptual y aplicada. Santiago, Chile: Universidad de Chile. Recuperado de http://repositorio.uchile.cl/handle/2250/120284.

Sen, A. (1999). Desarrollo y libertad. Barcelona, España: Planeta.

Sustainable Development Solutions Network [SDSN]. (2017). Cómo empezar con los ODS en las universidades. Una guía para las universidades, los centros de educación superior y el sector académico. Melbourne, Australia: Sustainable Development 


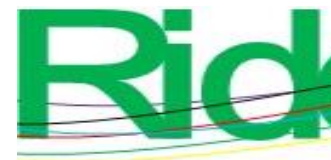

Revista Iberoamericana para la Investigación y el Desarrollo Educativo ISSN $2007-7467$

Solutions Network. Recuperado de http://reds-sdsn.es/wpcontent/uploads/2017/02/Guia-ODS-Universidades-1800301-WEB.pdf.

United Nations Educational, Scientific and Cultural Organization [Unesco]. (2019). Educational content up close. Examining the learning dimensions of Education for Sustainable Development and Global Citizenship Education. France: Organización de las Naciones Unidas para la Educación, la Ciencia y la Cultura. Retrieved from https://unesdoc.unesco.org/ark:/48223/pf0000372327.

Vallaeys, F. (2014). Manual de responsabilidad social universitaria. El modelo Ursula: estrategias, herramientas, indicadores. Unión de Responsabilidad Social Universitaria Latinoamericana. Recuperado de https://unionursula.org/publicacionesursula/.

Vallaeys, F., De la Cruz, C. y Sasia, P. M. (2009). Responsabilidad social universitaria: manual de primeros pasos. Ciudad de México, México: McGraw-Hill. Recuperado de https://publications.iadb.org/es/publicacion/14191/responsabilidad-socialuniversitaria-manual-de-primeros-pasos.

Wackerly, D., Mendenhall, W. y Scheaffer, R. (2010). Estadística matemática con aplicaciones (7. ${ }^{\mathrm{a}} \mathrm{ed}$.). Ciudad de México, México: Cengage Learning. Recuperado de https://www.google.com.mx/url?sa=t\&rct=j\&q=\&esrc=s\&source=web\&cd=\&cad=r ja\&uact=8\&ved=2ahUKEwibu9Px4JzsAhUbAp0JHQx4DE0QFjABegQIAhAC\&ur l=http $\% 3 \mathrm{~A} \% 2 \mathrm{~F} \% 2 \mathrm{Fwww}$. economia.unam.mx\%2Fbiblioteca\%2FPdf\%2FEstad\%25 C3\%25ADstica\%2520matem\%25C3\%25A1tica\%2520con\%2520aplicaciones\%252 07edi_MENDENHALL.pdf\&usg=AOvVaw32nJahvdekrkJ5DtiCbslP. 


\begin{tabular}{|l|l|}
\hline Rol de Contribución & Autor (es) \\
\hline Conceptualización & $\begin{array}{l}\text { Rosa Vega Cano Rogelio y Rivera Fernández (mismo grado de } \\
\text { contribución ambos autores) }\end{array}$ \\
\hline Metodología & $\begin{array}{l}\text { Rosa Vega Cano Rogelio y Rivera Fernández (mismo grado de } \\
\text { contribución ambos autores) }\end{array}$ \\
\hline Software & No aplica \\
\hline Validación & $\begin{array}{l}\text { Rosa Vega Cano Rogelio y Rivera Fernández (mismo grado de } \\
\text { contribución ambos autores) }\end{array}$ \\
\hline Análisis Formal & $\begin{array}{l}\text { Rosa Vega Cano Rogelio y Rivera Fernández (mismo grado de } \\
\text { contribución ambos autores) }\end{array}$ \\
\hline Investigación & $\begin{array}{l}\text { Rosa Vega Cano (principal) y Rogelio Rivera Fernández } \\
\text { (apoyo) }\end{array}$ \\
\hline Recursos & No aplica \\
\hline Curación de datos & $\begin{array}{l}\text { Rosa Vega Cano (principal) y Rogelio Rivera Fernández } \\
\text { (apoyo) }\end{array}$ \\
\hline $\begin{array}{l}\text { Escritura - Preparación del } \\
\text { borrador original }\end{array}$ & $\begin{array}{l}\text { Rosa Vega Cano Rogelio y Rivera Fernández (mismo grado de } \\
\text { contribución ambos autores) }\end{array}$ \\
\hline $\begin{array}{l}\text { Escritura - Revisión y } \\
\text { edición }\end{array}$ & $\begin{array}{l}\text { Rosa Vega Cano Rogelio y Rivera Fernández (mismo grado de } \\
\text { contribución ambos autores) }\end{array}$ \\
\hline Visualización & $\begin{array}{l}\text { Rosa Vega Cano Rogelio y Rivera Fernández (mismo grado de } \\
\text { contribución ambos autores) }\end{array}$ \\
\hline Supervisión & $\begin{array}{l}\text { Rosa Vega Cano Rogelio y Rivera Fernández (mismo grado de } \\
\text { contribución ambos autores) }\end{array}$ \\
\hline Administración de Proyectos & $\begin{array}{l}\text { Rosa Vega Cano Rogelio y Rivera Fernández (mismo grado de } \\
\text { contribución ambos autores) }\end{array}$ \\
\hline Adquisición de fondos & No aplica \\
\hline
\end{tabular}

